\title{
A sensitivity study of piezoresistive pressure sensor for robotic hand
}

\begin{abstract}
In this paper, we have studied and surveyed the field of robotic hand and the works that have been done in this area related to types of materials such as piezoresistive, piezoelectric and capacitive as well as a few types of pressure sensors. It indicates that piezoresistive pressure sensor is the best technique that can be used to implement a robotic hand for pick and place application. An adequate experiment of pressure sensor interfacing and calibration have been done in this paper. As a preliminary result of the works, output voltage (V) of the pressure sensor versus applied force input $(\mathrm{N})$ are presented. Furthermore, this framework can be used to derive a new approach of pressure sensor distribution on the robotic hand based on complex algorithm of controlling applied pressures.
\end{abstract}

Keyword: Pressure sensor; MEMS; Piezoresistive; Hand system; Dexterous manipulation 